\title{
MOLDANDO O FUTURO COM REPRESENTAÇÕES DO PASSADO EM CONSTANTE EVOLUÇÃO*
}

\author{
Neil Asher Silberman ${ }^{1}$
}

É para mim um grande prazer prestar homenagem ao professor Pedro Paulo Funari e procurar situar suas significantes contribuições para o desenvolvimento intelectual da arqueologia global, clássica e brasileira num contexto teórico e histórico. Em sua incansável e entusiasmada orientação a gerações de estudantes diplomados na Universidade Estadual de Campinas, em suas próprias fecundas publicações, e em sua participação e avaliação crítica das iniciativas contínuas do Congresso Arqueológico Mundial (Funari, 2006), Funari é reconhecido como um intelectual de liderança internacional na disciplina (Zarankin, 2014). Ainda assim, sua contribuição não tem sido meramente de preservar e passar adiante as técnicas reconhecidas da arqueologia e seu existente volume de conhecimento; sua façanha tem sido constantemente expandir as fronteiras da arqueologia no sentido dos temas sociais e práticas políticas raramente exploradas. Menciono aqui apenas algumas de suas publicações em inglês, sem englobar suas centenas de publicações em português. Mais notáveis são suas análises socio-econômicas da prática arqueológica brasileira (Funari, 2002), sobre a utilidade da arqueologia em documentar os crimes dos regimes ditatoriais latinoamericanos (Zarankin and Funari, 2008) e acerca do papel da educação arqueológica na formação da identidade brasileira contemporânea (Funari, 2000). Seu contínuo interesse teórico e ativismo no campo dos direitos humanos na América Larina e suas contribuições para a teoria cultural marcaram seu trabalho, não apenas no interior da academia, mas também como um intelectual público engajado.

Além disso, mesmo em sua pesquisa sobre sítios e assuntos arqueológicos específicos, Funari expandiu de maneira consistente as fronteiras disciplinares convencionais, focando-se no aparentemente inevitável emaranhado do presente e passado. Seja interrogando as interpretações conflitantes do caráter étnico do reino quilombola de Palmares no século XVII (homogeneamente africano ou multicultural?) (Funari, 2003); repensando e reavaliando o significado semiotico dos grafites de Pompeia e, através disso, reconsiderando

\footnotetext{
* Traduzido do inglês por Pedro Benedetti, Doutorando em História, USP.

1 Professor Associado, Universidade de Massachusetts Amherst, Massachusetts, Estados Unidos. E-mail: neil.silberman@gmail.com
} 
o desdém com o qual a cultura popular foi tratada nos estudos clássicos tradicionais (Funari, 1993); ou examinando as subordinações e dominação nas habitações domésticas em Buenos Aires desde o século XVIII (Pedro Paulo A. Funari and Zarankin, 2003), o trabalho de Funari e de seus colegas e orientandos não apenas expandiu o escopo do esforço arqueológico, mas trouxe sérios questionamentos de sua epistemologia básica. Com efeito, o debate da co-criação de uma memória coletiva compartilhada através de uma intensiva colaboração entre acadêmicos e o grande público (Funari e Carvalho, 2011) pode marcar o início de um movimento para além da arqueologia "pública" ou "da comunidade" para uma "herança pública" em evolução, a qual não privilegia uma metodologia particular em detrimento de outras.

Foi para mim uma grande honra visitar a UNICAMP em duas ocasiões e ver por mim mesmo o extraordinário encorajamento que Funari transmitiu a seus alunos em direção à contínua auto-crítica intelectual e à mudança metodológica. Seguindo a tradição progressista de Paulo Duarte, em virtude de quem o Laboratório de Arqueología Pública (LAP) recebeu seu nome (Funari e Carvalho, 2012), e na integração próxima entre os estudos ambientais e o trabalho de pesquisa arqueológico da universidade, Funari tem sido instrumental na criação de um centro transdisciplinar no qual a historiografia tradicional está sendo transformada num híbrido de ciências sociais, ecologia e humanidades. De fato, estou convencido de que isso é uma précondição para sustentar a utilidade das abordagens historiográficas no mundo imprevisível do Antropoceno. Os incessantes debates acerca do conceito de "autenticidade" na herança cultural (Gfeller, 2017); o papel das preocupações dos direitos humanos tanto em arqueologia e na prática de herança cultural (Silverman e Ruggles, 2007); e naturalmente o impacto da globalização nos contextos sociais, valores e significados dos lugares de herança cultural (Labadi e Long 2010). Estamos claramente em um período de transição profunda, tanto na academia quanto na sociedade global. A questão que se coloca, então, é: o que há para ser feito? A resposta, creio eu, reside numa reavaliação fundamental da relação entre a academia e a sociedade civil - e talvez ainda mais importante, numa reorientação temporal da narrativa histórica.

Acerca da primeira questão: a cocriação público-acadêmica das compreensões arqueológicas e históricas (as quais, como mencionado anteriormente, Funari e Carvalho começaram a explorar no contexto da Arqueologia Pública), estou convencido de que ainda há muito a ser explorado. A crescente influência da Ação de Pesquisa Participatória (PAR) em tão diversos campos como saúde pública, educação e desenvolvimento comunitário sugereum caminho para que os programas dirigidos por especialistas cedam lugar à colaboração com as comunidades direta ou indiretamente impactadas pela pesquisa acadêmica. Isso é de particular importância no domínio da arqueologia e da herança 
cultural, onde a pesquisa é levada a cabo em lugares específicos. Como Setha Low já apontou de maneira eloquente, o processo é o de placemaking2 e as abordagens binárias de cima para baixo e de baixo para cima podem ser superadas através da adoção de metodologias PAR (Low 2014). De fato, Funari fez com que fosse possível que eu e minha colega Angela Labrador trabalhássemos com os estudantes do LAP - NEPAM a fim de introduzir e experimentar as técnicas e conceitos básicos da PAR (Coherit Associates, 2014). O objetivo era conjugar ambos os fatos empíricos e expressões criativas num estudo de relevância para o campus da universidade. Para tal experimento, os estudantes serviram tanto como "especialistas" quanto como "comunidade" com o que era talvez o principal resultado pedagógico sendo $\mathrm{o}$ reconhecimento do quão indistintas essas categorias podem ser.

A segunda questão que eu acredito ser central para a transformação da arqueologia em seu papel de discurso público é uma reorientação básica da nossa perspectiva cronológico-temporal. A trajetória unidirecional da "flecha do tempo" do passado remoto para o presente vivido tem sido colocada em cheque tanto por seu reducionismo teleológico (Landa, 1997) quanto por sua forma literária distintiva (White, 1987). Eu acrescentaria ainda que sua valência política como inerente auto-justificativa política - seja das elites, seja dos dissidentes e críticos - está implícita em suas formas tradicionais (Silberman, 1996). Ao colocar uma fronteira ontológica entre "nossa" história e o "mito deles", ela cria a base para a continuidade do conflito entre "nós" e "eles" (Silberman, 2013). Nesse sentido, o prudente aviso de Bruce Trigger a respeito da instrumentalidade política inerente à arqueologia como sendo inevitavelmente nacionalista colonialista ou imperialista (Trigger, 1984), não pode ser mais ignorada. Pois se um futuro compartilhado e inclusivo é o objetivo da pesquisa arqueológica, da arqueologia comunitária ou pesquisa de ação participativa, a forma narrativa de expressão historiográfica deve transcender das injustiças e a cegueira do presente ao explicitamente representar tal objetivo em sua narrativa, de modo que a visão de um futuro imaginado, de maneira contra-intuitiva mas poderosa, anteceda a reconstrução do passado (cf. Avanessian e Malik, 2016).

Há uma conexão clara entre essas duas preocupações - da co-criação historiográfica e da construção narrativa - na cristalização do que poderíamos chamar de "herança pública". Certamente, a própria estrutura do LAPNEPAM na UNICAMP e os interesses de pesquisa de sua faculdade e de seus estudantes, abrangendo desde a ciência ecológica até a cultura popular,

\footnotetext{
2 O conceito foi cunhado em 1980 nos EUA para designar um processo de planejamento, criação e gestão de espaços públicos, que estimula uma maior interação entre as pessoas e propõe a transformação dos pontos de encontro de uma comunidade em lugares mais agradáveis e atrativos (N. do T)
} 
estudos de mídia, gerenciamento de recursos culturais e epigrafia clássica representa uma prática interdisciplinar emergente que se endereça simultaneamente ao emaranhado do passado, presente e a incerteza do futuro. E é algo que não tem a pretensão de, dadas suficientes verbas, participantes e equipamentos de escavação, descobrir de uma vez por todas um passado rankiano "como realmente aconteceu" - " wie es eigentlich gewesen". Ocorreume diversas vezes em minha carreira de escrever sobre a história e a política da arqueologia e herança cultural que a evolução de uma narrativa historiográfica aceita se deve somente parcialmente à acumulação de fatos empíricos. Conquanto a acumulação gradual de evidências empíricas seja a marca registrada de toda a produção acadêmica pós-iluminismo e tem sido utilizada para justificar a natureza "científica" do estudo histórico, a evidência histórica raramente é auto-explicativa e tem de ser interpretada. O contexto para essa interpretação tem sempre sido a gama de questões socioeconômicas de quem interpreta, qualquer que seja a época em que ele ou ela viva. Fatos são indubitavelmente essenciais, mas eles não contam toda a história, ou mesmo carregam um significado intrínseco. Nesse sentido, a historiografia deve ser considerada como um discurso público que desenha a rota de uma sociedade para o futuro, mais do que documenta objetivamente seu passado (Silberman, 2013a).

O produto da historiografia, eu argumentaria, é o processo da própria historiografia. E esse processo toma forma num presente em constante transformação, tornado ainda mais imprevisível na alvorada do Antropoceno. Com efeito, a empolgação com novas compreensões ecológicas e descobertas históricas deriva de sua justaposição com as condições socioeconômicas em constante mutação, bem como desafios ambientais inesperados. Como discurso público, a História, a Arqueologia e a herança cultural oferecem uma oportunidade para que o futuro continue aberto a trajetórias alternativas e não simplesmente explicam por que a atual disposição de poder socioeconômico e condições ambientais eram historicamente inevitáveis.

As condições mudaram dramaticamente desde a minha última visita ao Brasil em 2014. E ao redor do mundo, o incansável processo da mudança climática e o predomínio do neonacionalismo xenofóbico e do fundamentalismo religioso oferecem um novo contexto no qual o passado deve ser visto. É um tempo propício para que a interação íntima entre entendimentos do passado e antecipações do futuro sejam reconhecidos e incorporados no processo historiográfico. Quer arqueológica, literária ou performativa, a História é o que a História faz na era em que se vive. E o fazer de uma constante obrigação social para a academia e igualmente para o público geral. Estamos no limite de uma reorientação copernicana na reflexão histórica, na qual o futuro, não o passado, é o centro do nosso universo cronológico. O imperativo ético é 
reconhecer que não há respostas finais para questões históricas. A responsabilidade crucial de todas as disciplinas historiográficas - muito bem alinhadas com o encorajamento que funari passa aos seus estudantes - é que a pesquisa histórica e arqueológica é e deve sempre ser um processo contínuo de reflexão e debate social e político - com todas suas fantásticas consequências para o reconhecimento de direitos humanos, tolerância de coexistência cultural e intensificação da dignidade humana.

\section{Referências}

AVANESSIAN, Armen, e MALIK, Suhail. The Speculative Time Complex. In The Time Complex. Post-Contemporary, 2016, 5-56. Miami, Florida: [NAME] Publishers.

Coherit Associates. "Values-Based Heritage Approaches for Sustainable Development." 2014. http://coherit.com/projects.html.

FUNARI, Pedro Paulo Abreu. Graphic Caricature and the Ethos of Ordinary People at Pompeii. Journal of European Archaeology 1 (2): 1993, 133-50. $182-85$.

. Archaeology, Education and Brazilian Identity. Antiquity 74 (283): 2000,

- Class Interests in Brazilian Archaeology. International Journal of Historical Archaeology 6 (3): 2002, 209-16.

Conflict and the Interpretation of Palmares, a Brazilian Runaway Polity. Historical Archaeology 37 (3): 2003, 81-92.

. The World Archaeological Congress from a Critical and Personal Perspective. Archaeologies, 2006, no. 1:73.

FUNARI, Pedro Paulo, CARVALHO,Aline Vieira. Inclusion in Public Archeology in Brazil: Remarks on Collaborative Practices. Archaeologies 7 (3): 2011, 554-73.

. Universidades, Arqueologia e Paulo Duarte. Revista do Museu de Arqueologia e Etnologia 0 (22): 2012, 89-96.

FUNARI, Pedro Paulo A., ZARANKIN., Andrés. Social Archaeology of Housing from a Latin American Perspective: A Case Study. Journal of Social Archaeology 3 (1): 2003, 23-45. 
GFELLER, Aurélie Élisa. The Authenticity of Heritage: Global Norm-Making at the Crossroads of Cultures. The American Historical Review 122 (3): 2017, 758791.

LABADI, Sophia, LONG, Colin. Heritage and Globalisation. Milton Park, Abingdon, Oxon, England; New York, NY: Routledge, 2010.

LANDA, Manuel De. A Thousand Years of Nonlinear History. First Edition edition. New York: Zone Books, 1997.

LOW, Setha M. Spatializing Culture: An Engaged Anthropological Approach to Space and Place. In The People, Place and Space Reader, 2014.

SILBERMAN, Neil A. Promised Lands and Chosen Peoples: The Politics and Poetics of Archaeological Narrative. In Nationalism, politics, and the Practice of Archaeology.Cambridge University Press, 1996.

. Heritage Interpretation as Public Discourse. In Understanding Heritage. Ed. Albert, Marie-Theres / Bernecker, Roland / Rudolff, Britta, 21-33, $2013 \mathrm{a}$. Berlin: De Gruyter.

. The Tyranny of Narrative: History, Heritage, and Hatred in the Modern Middle East. Journal of Eastern Mediterranean Archaeology and Heritage Studies 1 (2): 2013b, 175-184.

SILVERMAN, Helaine, e D. Fairchild RUGGLES. Cultural Heritage and Human Rights. New York; London: Springer, 2007.

TRIGGER, Bruce G. Alternative Archaeologies: Nationalist, Colonialist, Imperialist. Man 19 (3): 1984, 355-70.

WHITE, Hayden. 1987. The Content of the Form: Narrative Discourse and Historical Representation. Baltimore: The Johns Hopkins University Press.

ZARANKIN, Andrés. FUNARI, Pedro Paulo A. (Historical Archaeology). In Encyclopedia of Global Archaeology, edited by Claire Smith, 2937-39, 2014. New York, NY: Springer New York.

ZARANKIN, Andrés, FUNARI, Pedro Paulo Abreu. Eternal Sunshine of the Spotless Mind': Archaeology and Construction of Memory of Military Repression in South America (1960-1980)." Archaeologies 4 (2): 2008. 310-27. 